\title{
RNA-seq liver transcriptome analysis reveals an activated MHC-I pathway and an inhibited MHC-II pathway at the early stage of vaccine immunization in zebrafish
}

\author{
Dahai Yang, Qin Liu*, Minjun Yang, Haizhen Wu, Qiyao Wang, Jingfan Xiao and Yuanxing Zhang*
}

\begin{abstract}
Background: Zebrafish (Danio rerio) is a prominent vertebrate model of human development and pathogenic disease and has recently been utilized to study teleost immune responses to infectious agents threatening the aquaculture industry. In this work, to clarify the host immune mechanisms underlying the protective effects of a putative vaccine and improve its immunogenicity in the future efforts, high-throughput RNA sequencing technology was used to investigate the immunization-related gene expression patterns of zebrafish immunized with Edwardsiella tarda live attenuated vaccine.

Results: Average reads of 18.13 million and 14.27 million were obtained from livers of zebrafish immunized with phosphate buffered saline (mock) and E. tarda vaccine (WED), respectively. The reads were annotated with the Ensembl zebrafish database before differential expressed genes sequencing (DESeq) comparative analysis, which identified 4565 significantly differentially expressed genes (2186 up-regulated and 2379 down-regulated in WED; $p<0.05)$. Among those, functional classifications were found in the Gene Ontology database for 3891 and in the Kyoto Encyclopedia of Genes and Genomes database for 3467. Several pathways involved in acute phase response, complement activation, immune/defense response, and antigen processing and presentation were remarkably affected at the early stage of WED immunization. Further GPCR analysis confirmed that the genes encoding the factors involved in major histocompatibility complex (MHC)-I processing pathway were up-regulated, while those involved in MHC-II pathway were down-regulated.
\end{abstract}

Conclusion: These data provided insights into the molecular mechanisms underlying zebrafish immune response to WED immunization and might aid future studies to develop a highly immunogenic vaccine against gram-negative bacteria in teleosts.

\section{Background}

Zebrafish (Danio rerio) is a well-established vertebrate model of human development and hematopoiesis [1]. However, as the aquaculture industry grows to meet the needs of an expanding global population, there has been a rapid increase in the research of teleost physiology and immunology. The zebrafish model system has proven to be a useful tool for studying infectious diseases that are natural threats to fish species of important human food sources, such as rock bream (Oplegnathus fasciatus) and

\footnotetext{
*Correspondence: qinliu@ecust.edu.cn; yxzhang@ecust.edu.cn

State Key Laboratory of Bioreactor Engineering, East China University of Science and Technology, 130 Meilong Road, Shanghai 200237, China
}

turbot (Psetta maxima) [2]. Several studies of the zebrafish immune system have provided initial insights into host-microbe interactions with both pathogens and commensals [3,4], and the molecular processes mediating clearance of pathogenic infections [5-7]. Not surprisingly, many features of the zebrafish defense responses following pathogen invasion resembled those of other mammals, including humans [7-9].

The adult zebrafish has been used in a few studies to investigate new vaccines against specific pathogenic species. For example, Novoa et al. [10] studied the efficacy of a vaccine derived by reverse genetics against viral hemorrhagic septicemia virus in fish by using the 
zebrafish as a model system, and revealed that the vaccine was protective even at low temperatures. In addition, Cui et al. [11] used zebrafish to study an attenuated live Mycobacterium marinum vaccine aimed at reducing mycobacteriosis in freshwater and marine fish. Finally, Xiao et al. [12] developed an edwardsiellosis zebrafish model to screen attenuated live Edwardsiella tarda vaccine candidates in order to identify those most highly effective for subsequent development for industry use. However, so far, no work involves the immunerelated pathways underlying the zebrafish response to vaccination.

In order to design a novel and effective vaccine, it is essential to gain a comprehensive understanding of the immune responses elicited in host upon vaccination. To date, most of the studies of the teleost immune system have focused on head kidney or/and spleen [13]. However, the vertebrate liver has recently been recognized as an essential immune organ [14-16], accommodating a variety of cell types $[14,17]$, including those primarily involved in immune activities. Since the liver receives blood from both the systemic circulation and the intestine, it is exposed to a wide array of antigens. Therefore, its immune-related cellular components can manifest a broad range of immune reactions $[14,18]$. For example, the liver lymphocyte population includes both innate immune cells (such as the macrophages and natural killer cells) and adaptive immune cells (such as conventional $\mathrm{T}$ lymphocytes [14,19] that recognize and respond to antigenic peptides presented by the major histocompatibility complex (MHC)-I or -II [18,20]). As such, different infectious pathogens would be expected to induce distinctive profiles of immune responses in the liver [21], which might be manipulated to create specific and effective therapeutic strategies.

Several methods exist by which to determine the comprehensive transcriptomic profile of a pathogen-specific immune response, including microarray and quantitative real-time PCR [22,23]. However, the high-throughput RNA sequencing (RNA-seq) technology offers several advantages over the other profiling applications. Not only is RNA-seq independent on predefined probes, which facilitates the discovery of new transcript variants, but the sequence platform also produces low background noise, which allow for distinction between closely homologous genes and detection of weakly expressed transcripts [24]. In addition, concurrent advances in the bioinformatic algorithms used to analyze the RNA-seq data have allowed for better interpretation of the whole transcriptomic profile and provided further insights into complex molecular processes. The RNAseq approach has already been successfully applied to several infectious disease models of zebrafish [25-27], including zebrafish embryo infected with Salmonella [26], and adult zebrafish and embryos infected by Mycobacteria [25,28]. In addition, other fish species infection models have been subjected to RNA-seq analysis, including large yellow croaker (Larimichthys crocea) infected by Aeromonas hydrophila [29] and Japanese seabass (Lateolabrax japonicus) infected by Vibrio Harveyi [30], but the overall immune-related transcription profiles have differed among species [25-30]. No reports exist in the literature of RNA-seq technology used to analyze the changes in an infected fish transcriptome profile induced upon vaccine treatment.

Edwardsiellosis, caused by the gram-negative Edwardsiella tarda, is currently one of the most economically disastrous infectious diseases affecting the global aquaculture industry [31]. E. tarda displays polymorphic phenotypes and has a broad range of hosts from aquatic invertebrates to higher vertebrates, including birds, reptiles, mammals, and even humans [31]. In developing a putative live attenuated vaccine against edwardsiellosis, Xiao et al. [12] constructed an E. tarda mutant (WED) with low residual virulence. Although the mutant was capable of inducing robust protection in zebrafish and turbot, the antibody titers detected in sera were relatively low. By thoroughly understanding the immunemechanism of zebrafish induced by the putative live attenuated vaccine, a more immunogenic vaccine may be able to be generated. To this end, we performed a comparative gene transcription analysis of livers from mock-immunized and WED-immunized zebrafish using RNA-seq technology to investigate their differential transcriptsomic profile. Furthermore, 12 genes associated with $\mathrm{MHC}$ antigen processing were analyzed by qPCR and the results revealed an activated MHC-I pathway and an inhibited MHC-II pathway during the early stage of vaccine immunization. It was prompted that WED conferred a robust protection in zebrafish by eliciting an effective cell immunity via the MHC-I pathway.

\section{Results}

\section{RNA-seq of liver transcriptome}

To better understand the early stage immune response of zebrafish immunized with WED, six Solexa cDNA libraries were constructed from the livers of mockimmunized and WED-immunized zebrafish (Additional file 1). Biological replicates were pooled to make representative samples for deep sequencing analysis. Across the two groups of triplicate data, after normalization of the generated 95 bp PE raw reads, 15,683,828, $13,040,780$ and $25,660,654$ reads were obtained from C1-C3, and 16,306,312, 15,589,848 and 10,906,906 reads from V1-V3, respectively. To assess the quality of sequencing, the reads were mapped to the zebrafish reference genome. From the reads of each group, successful 
mapping occurred for 10,823,266 (C1), 9,584,828 (C2), $18,321,987$ (C3), 12,209,418 (V1), 11,675,593 (V2) and $8,605,104$ (V3) reads. However, 4,860,562, 3,455,952 and $7,338,667$ unmapped reads were generated from C1-C3, while 4,096,894, 3,914,255 and 2,301,802 unmapped reads were found in V1-V3, respectively; we plan to conduct de novo analysis of these unmapped reads to generate a better reference of immune-relevant genes in zebrafish.

\section{Analysis of differential expression among WED- and mock-immunized zebrafish liver}

To identify the differentially expressed genes, the transcriptome data of zebrafish liver from two days after WED immunization and mock immunization were analyzed by using the DESeq package in $\mathrm{R}$ software [32]. The criteria of a two-fold or greater change in expression and $p$-value $<0.05$ (cut off at $5 \%$ FDR) were chosen to determine significantly up-regulated or downregulated genes following immunization. The magnitude distribution of the significantly changed genes was illustrated by MA plot analysis (Figure 1). Using these criteria, a total of 4565 genes were significantly differentially expressed greater than two-fold, including 2186 up-regulated genes and 2379 down-regulated genes (Additional file 2). Annotation of the differentially expressed genes was achieved through BLASTN similarity searches against the Ensembl zebrafish RefSeq mRNA database (Version danRer 7, Additional file 2).

To perform an unbiased annotation of the functions of the differentially expressed genes identified by DESeq

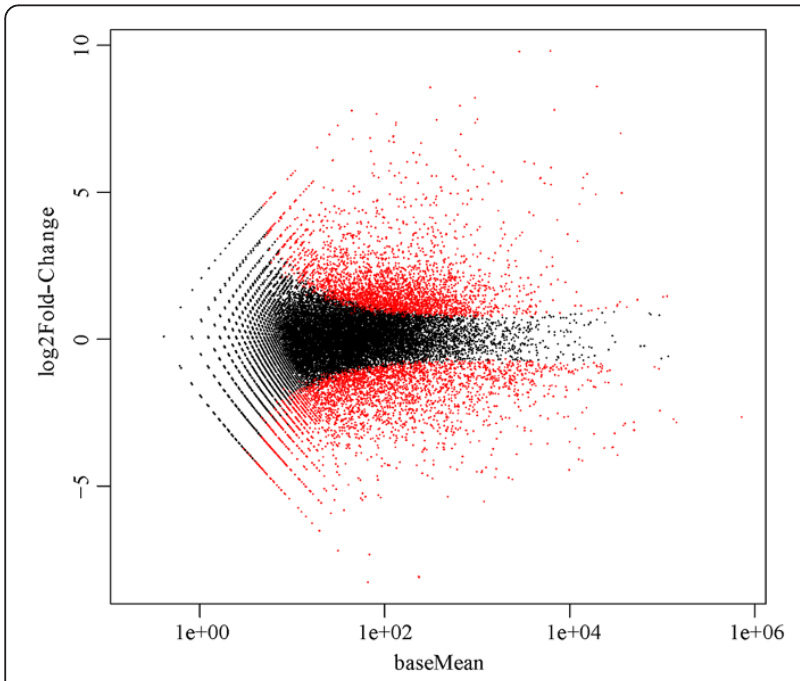

Figure 1 MA plot of differentially expressed genes identified in WED-immunized and mock-immunized zebrafish livers. Data represent individual gene responses plotted as $\log _{2}$ fold-change versus baseMean fold-change $>2$ ( $p$-values $<0.05)$, with a negative change representing the down-regulated genes and a positive change representing the up-regulated genes. analysis, GO analysis of differentially expressed genes was carried out by two bioinformatics tools, DAVID and BiNGO plugin (Additional file 3). Among the 4565 differentially expressed genes, DAVID provided functional annotation for 3891 genes. GO annotated differentially expressed genes mainly belonged to the three functional clusters (biological process, cellular component, and molecular function), and were distributed among more than 70 categories (Additional file 3). The differentially expressed genes in the cluster of biological processes were found to be mainly related to stimulus response, immune response, regulation of immune system process, and regulation of development process (Figure 2).

To identify the biological pathways that are active in the zebrafish at the early stages of WED immunization, 4565 differentially expressed genes were mapped to canonical signaling pathways found in KEGG. A total of 3467 genes were mapped to 14 statistically significant categories $(p<0.05$; Table 1$)$. Protein processing in ER was represented by 73 up-regulated and 8 downregulated genes. There were also a statistically significant amount of mapped genes for other major antigen processing-related pathways, such as those mediated by "proteasome" and "protein export" pathway, to indicate the vital role of antigen processing and presentation activated by WED immunization at the early stage in zebrafish liver, which would elicit the specific immune responses required in the restoration of homeostasis.

In general, based on the results from GO analysis (by BiNGO plugin) and KEGG pathway analysis, the upand down-regulated genes that were highly related to immune response of fish after WED immunization, significantly grouped into acute phase response (APR), complement activation, immune/defense response, and antigen processing and presentation pathway.

\section{The acute phase response is conserved in zebrafish liver following WED immunization}

Most of the conserved acute phase response genes were significantly differentially expressed following WED immunization (Table 2). This set of genes encoded the major APPs (e.g. serum amyloid A (SAA), C-reactive protein (CRP), and serum amyloid P (SAP)), the minor and intermediate APPs (e.g. fibrinogen, plasminogen, plasminogen activator inhibitor, antitrypsin, ceruloplasmin, hemopexin, haptoglobin, and ferritin), the negative APPs (apolipoprotein A-IV and alpha-2-HS-glycoprotein), several complement components, and ion-binding and transporting proteins. Many of the APPs were up- or down-regulated greater than five-fold, suggesting that induction of APPs in zebrafish liver likely plays an important role in host defense stimulated by WED vaccine. Similar subcategories of APPs were also found to be differentially expressed in previous microarray-based studies of early 


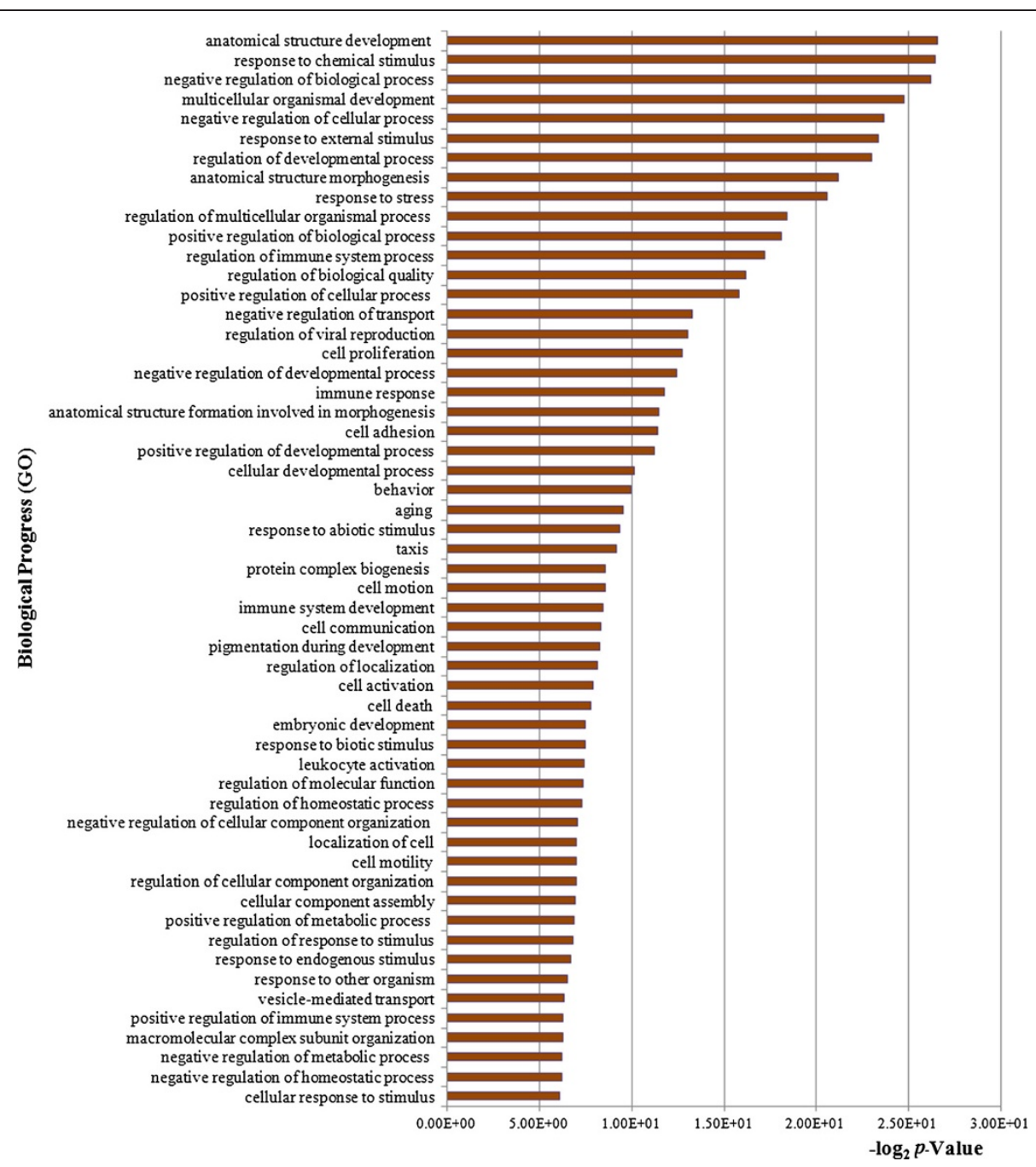

Figure 2 Significantly up-regulated genes assigned to GO biological process categories.

stage immune response to bacterial infection in rainbow trout [33] and catfish [34], indicating the conservation of the vast majority of APPs among teleost fish. The major APPs, two CRP-like proteins (CRP and SAP) and SAA, were induced to up-regulated their expressions by 9.7-fold, 2.1-fold and 883-fold, respectively, in the WED-immunized zebrafish liver, emphasizing their importance in teleost innate immune response. In WED-immunized zebrafish, both the apolipoprotein AIV, a fatty acid binding protein involved in extracellular and intracellular lipid transporting, and alpha-2-HS -glycoprotein were decreased by 3.0 -fold and 11.8 -fold, respectively. The decrease of apolipoprotein A-IV was consistent to previous report in starved zebrafish liver [35]. However, the functions of these two negative APPs in immune response remain unknown.

Traditionally, complement has been considered as a supportive first line of defense against microbial intruders
[36]. In WED-immunized zebrafish liver, three isoforms of complement C3, and the complement C4, C2, C7 and C9 were remarkably up-regulated. The $\mathrm{C} 1$ inhibitor and the C1q were up-regulated and down-regulated, respectively (Table 2).

Hepatocytes, which account for $80 \%$ of the liver mass [21], are the primary site of synthesis for all the genes involved in ion-binding and transporting [33,35,37]. In the RNA-seq data, more than 20 differentially expressed genes involved in ion-binding and transporting were strongly induced in zebrafish liver upon WED immunization (Table 2). These included haptoglobin, hemopexin, ceruloplasmin, transferrin receptor 2 , ATPase, and $\mathrm{Cu}^{2+}$ transporting alpha polypeptide. Intelectin [33], which is involved in iron homeostasis, binding and transport, was one of the most up-regulated genes (129-fold) in the ionbinding and transporting category; However, the functions of intelectin in the contexts of normal iron metabolism 
Table 1 Statistically significant KEGG classifications of differentially expressed genes

\begin{tabular}{lccc}
\hline KEGG subcategories & Counts & $\%^{*}$ & $\boldsymbol{p}$-value \\
\hline Ribosome & 70 & 1.53 & $9.60 \mathrm{E}-14$ \\
Proteasome & 35 & 0.77 & $1.80 \mathrm{E}-13$ \\
Protein processing in endoplasmic reticulum & 81 & 1.77 & $2.27 \mathrm{E}-10$ \\
Arginine and proline metabolism & 27 & 0.59 & $2.26 \mathrm{E}-04$ \\
Primary bile acid biosynthesis & 10 & 0.22 & $8.23 \mathrm{E}-04$ \\
Fructose and mannose metabolism & 18 & 0.39 & $2.44 \mathrm{E}-03$ \\
Glycolysis/Gluconeogenesis & 27 & 0.59 & $3.47 \mathrm{E}-03$ \\
N-Glycan biosynthesis & 22 & 0.48 & $3.71 \mathrm{E}-03$ \\
Glycine, serine and threonine metabolism & 15 & 0.33 & $1.64 \mathrm{E}-02$ \\
Galactose metabolism & 13 & 0.28 & $2.23 \mathrm{E}-02$ \\
Histidine metabolism & 12 & 0.26 & $4.02 \mathrm{E}-02$ \\
Peroxisome & 27 & 0.59 & $4.50 \mathrm{E}-02$ \\
Propanoate metabolism & 13 & 0.28 & $4.84 \mathrm{E}-02$ \\
Protein export & 13 & 0.28 & $4.96 \mathrm{E}-02$ \\
\hline
\end{tabular}

* indicates the percentage of genes in each pathway from 3467 genes mapped to KEGG.

and disease defense in zebrafish need to be further clarified. Members of the transferrin and ferritin families were significantly affected to result in obvious up- and downregulation in zebrafish liver by WED immunization (Table 2). Leukocyte cell-derived chemotaxin 2 (LECT2) [35], originally named for its possible neutrophil chemotactic activity in vitro, was strongly induced by 222.8 -fold in WED-immunized group, but its function in zebrafish remains unknown.

\section{WED immunization induces defense responses and signaling transduction pathways}

Functional annotation of significantly differentially expressed genes in zebrafish liver was performed to define the transcriptome profile more precisely. GO classification (Figure 2 and Additional file 3) indicated that immune/ defense response-related genes were enriched, specifically under GO terms "response to chemical stimulus", "regulation of immune system process", and "immune response". Toll-like receptors (TLRs) detected the presence of pathogens and triggered an innate immune response, and several of the differentially expressed genes from WED-immunized liver mapped to the TLR signaling pathways (Figure 3). TLR signaling has been remarkably conserved throughout evolution, and it can mediate immune responses to all types of pathogens and promote secondary disease. In zebrafish, the pathogenesis of M. marinum, Staphylococcus aureus, and Aeromonas salmonicida has been shown to involve TLR signaling [25,35]. To further investigate the function of TLR $5 \mathrm{a} / \mathrm{b}$ that elicited the immune response in zebrafish embryo, assessment of tlr5a and $t l r 5 b$ by morpholino-mediated knockdown followed by flagellin stimulation clearly demonstrated TLR5-dependent gene activation of $m m p 9$, cxcl-C1c, and irak3, which suggested that the activation of TLR5 pathway can induce the expression of inflammatory mediators as well as the feedback control of the innate immune response [38]. The functional investigation of TLR4 was also performed in a zebrafish embryo model, which suggested that the zebrafish TLR4 orthologs would negatively regulate the MyD88-dependent NF- $\mathrm{BB}$ activation by sequestering the TLR adaptors and indicated that the existence of a TLR would negatively regulate TLR signaling upon engagement with its specific ligand [39]. In humans and rodents, TLR-mediated signals in liver are associated with infection-induced granulomatous inflammation and ischemia-reperfusion injury, and can mediate liver regeneration processes [40]. In WED-immunized zebrafish liver, 21 differentially expressed genes mapped to various TLR pathways (Figure 3), including the TLR5, TLR8, TLR18 and TLR21 subcategories, which are not only expressed on the outer membrane of immune cells but also on endosome-lysosome membranes. TLR5 expression was up-regulated by 36.4-fold, indicating that it played a key role during the early stage of WED vaccination.

Immunity is a complex process of tightly controlled signals that involve a broad array of receptors, cytokines, enzymes, signal transducers, transcription factors, and other functional proteins. In our study, WED immunization increased dramatically the expression of cytokine genes related to the Jak-STAT, MAPK, TGF- $\beta$, apoptosis and VEGF signaling pathways. Therefore, WED-induced gene expression in zebrafish liver might facilitate protection against E. tarda by activating these pathways. Similar results were obtained in a previous study of large yellow croaker spleen during A. hydrophila infection [29]. Since the majority of the differentially expressed genes in these signaling pathways were up-regulated in our study, it is possible that the WED immunization of zebrafish is capable of triggering a vigorous adaptive immune response.

\section{WED immunization induces the antigen processing and presentation pathway}

A large number of differentially expressed genes with functions in protein transportation, modification and degradation were up- or down-regulated in the zebrafish liver following WED immunization (Table 3), indicating these genes were likely connected to the degradation and processing of antigens for MHC class I and II molecules. Most of the differentially expressed genes related to MHC-I antigen processing pathways were significantly up-regulated, including the ER-resident chaperone calreticulin, calnexin, endoplasmin (grp94), TAP binding protein, proteasome activator (PA28), the heat shock proteins superfamilies, and cathepsin L. Meanwhile, the typical MHC-II processing pathway component, 
Table 2 Significantly differentially expressed genes in acute phase response

\begin{tabular}{|c|c|c|c|c|c|c|}
\hline Gene name & Accession number & Description & RPKM-C & RPKM-V & Fold-change & $p$-value \\
\hline ahsg & ENSDARG00000069293 & Alpha-2-HS-glycoprotein & 554.80 & 55.81 & -11.84 & $7.26 \mathrm{E}-50$ \\
\hline apoa4 & ENSDARG00000040298 & Apolipoprotein A-IV & 266.33 & 164.60 & -2.09 & $2.21 \mathrm{E}-04$ \\
\hline tfre & ENSDARG00000012552 & Transferrin receptor (p90, CD71) & 23.29 & 13.29 & -2.27 & $1.18 \mathrm{E}-04$ \\
\hline$c l a b$ & ENSDARG00000044612 & Complement component 1, q subcomponent & 6.06 & 3.14 & -2.23 & $6.06 \mathrm{E}-03$ \\
\hline plg & ENSDARG00000023111 & Plasminogen & 64.66 & 37.99 & -2.18 & $6.73 \mathrm{E}-04$ \\
\hline aerping1 & ENSDARG00000058053 & C1 inhibitor & 15.69 & 6.71 & -2.93 & $5.49 \mathrm{E}-05$ \\
\hline$c 2$ & ENSDARG00000019772 & Complement component 2 & 24.57 & 52.88 & 2.06 & $2.99 \mathrm{E}-10$ \\
\hline si:dkey-8 k3.2 & ENSDARG00000038424 & Complement C4 & 323.79 & 978.12 & 2.07 & 8.93E-13 \\
\hline$c f b$ & ENSDARG00000055278 & Complement factor B & 727.97 & 1914.46 & 2.09 & $3.38 \mathrm{E}-03$ \\
\hline apcs & ENSDARG00000045089 & Serum amyloid P component & 309.74 & 872.88 & 2.16 & $1.64 \mathrm{E}-04$ \\
\hline$c p$ & ENSDARG00000010312 & Ceruloplasmin (Fragment) & 633.50 & 1737.21 & 2.19 & $2.74 \mathrm{E}-03$ \\
\hline c9 & ENSDARG00000016319 & Complement component C9 & 18.78 & 48.27 & 2.28 & $4.90 \mathrm{E}-157$ \\
\hline$c f h / 2$ & ENSDARG00000056778 & Complement factor $\mathrm{H}$, like 2 & 11.76 & 38.65 & 2.28 & $6.44 \mathrm{E}-07$ \\
\hline tfr2 & ENSDARG00000089980 & Transferrin receptor 2 & 10.84 & 34.09 & 2.51 & $2.58 \mathrm{E}-04$ \\
\hline fga & ENSDARG00000020741 & Fibrinogen alpha chain & 375.41 & 1118.34 & 2.55 & $4.87 \mathrm{E}-05$ \\
\hline c3a & ENSDARG00000012694 & Complement component c3a & 1305.16 & 4584.02 & 2.77 & $1.21 \mathrm{E}-03$ \\
\hline serpina7 & ENSDARG00000087143 & Antitrypsin & 466.41 & 1720.58 & 3.05 & $1.18 \mathrm{E}-05$ \\
\hline c3 & ENSDARG00000043719 & PREDICTED: complement C3-H2-like & 154.97 & 692.50 & 3.44 & 1.19E-08 \\
\hline serpine1 & ENSDARG00000056795 & Plasminogen activator inhibitor type 1 & 13.14 & 68.84 & 4.38 & $6.20 \mathrm{E}-14$ \\
\hline cebpb & ENSDARG00000042725 & CCAAT/enhancer binding protein, beta & 37.33 & 202.58 & 4.42 & $4.58 \mathrm{E}-13$ \\
\hline fgg & ENSDARG00000037281 & Fibrinogen, gamma polypeptide & 154.90 & 965.71 & 5.39 & $6.08 \mathrm{E}-15$ \\
\hline fth1a & ENSDARG00000015551 & Ferritin & 180.30 & 1233.87 & 5.45 & $9.51 \mathrm{E}-14$ \\
\hline$f 2 r$ & ENSDARG00000060012 & Coagulation factor II (Thrombin) receptor & 0.38 & 3.11 & 6.57 & $6.63 \mathrm{E}-05$ \\
\hline si:ch211-234p6.7 & ENSDARG00000071456 & C-reactive protein & 0.37 & 3.41 & 9.71 & $2.46 \mathrm{E}-04$ \\
\hline atp7a & ENSDARG00000003699 & ATPase, $\mathrm{Cu}++$ transporting & 0.21 & 3.99 & 13.09 & $2.38 \mathrm{E}-06$ \\
\hline hpx & ENSDARG00000051912 & Hemopexin & 192.84 & 7565.38 & 31.36 & $3.57 E-17$ \\
\hline $\operatorname{tr} 5 b$ & ENSDARG00000052322 & Toll-like receptor 5 & 2.17 & 98.99 & 36.41 & 7.93E-62 \\
\hline lygl1 & ENSDARG00000056874 & Lysozyme & 11.85 & 641.71 & 41.37 & $1.70 \mathrm{E}-41$ \\
\hline c7 & ENSDARG00000057121 & Complement component 7 & 6.38 & 435.85 & 63.02 & 4.13E-36 \\
\hline$i t \ln 3$ & ENSDARG00000003523 & Intelectin 3 & 52.98 & 8467.17 & 129.02 & $3.93 E-33$ \\
\hline lect2| & ENSDARG00000033227 & Leukocyte cell-derived chemotaxin 2 & 8.75 & 2597.52 & 222.85 & $8.92 \mathrm{E}-56$ \\
\hline$h p$ & ENSDARG00000051890 & Haptoglobin & 14.36 & 7332.05 & 387.28 & $1.97 E-30$ \\
\hline saa & ENSDARG00000045999 & Serum amyloid protein $\mathrm{A}$ & 1.24 & 1248.94 & 883.07 & $2.56 \mathrm{E}-66$ \\
\hline
\end{tabular}

Identification of all differentially expressed genes was based on $p<0.05$. A $p$-value $<0.05$ indicated that the gene was significantly altered in WED immunized fish with respect to mock-immunized fish. The absolute value of "Fold-change" is the magnitude of up- or down-regulation for each gene/homolog after WED immunization. Fold-change $>2$ indicates up-regulation, and $<-2$ indicates down-regulation.

lysosomal membrane glycoprotein 2 (lamp2), was downregulated (by 4.1-fold) in zebrafish liver after live attenuated vaccine immunization.

Up-regulated genes with established roles in immune responses comprised another large functional category, indicating that active immune-surveillance, immune signaling, and immune cell activation were triggered in the WED-immunized zebrafish liver, like the MHC-I ZE protein (by 3.09-fold). However, the MHC class II DAB, MHC class II beta chain, MHC class II invariant chain
(CD74 molecule), MHC class II transactivator (CIITA), and cathepsin $\mathrm{S}$ were down-regulated at this stage (Table 3).

To further explore the immune response profiles induced by WED immunization to the level of a single pathway, we used the KAAS web-based pathway analysis program. KEGG analysis was performed to identify genes involved in phagosome and antigen processing and presentation pathways (Figure 4). In the phagosome pathway, 35 genes were identified as strongly up- 


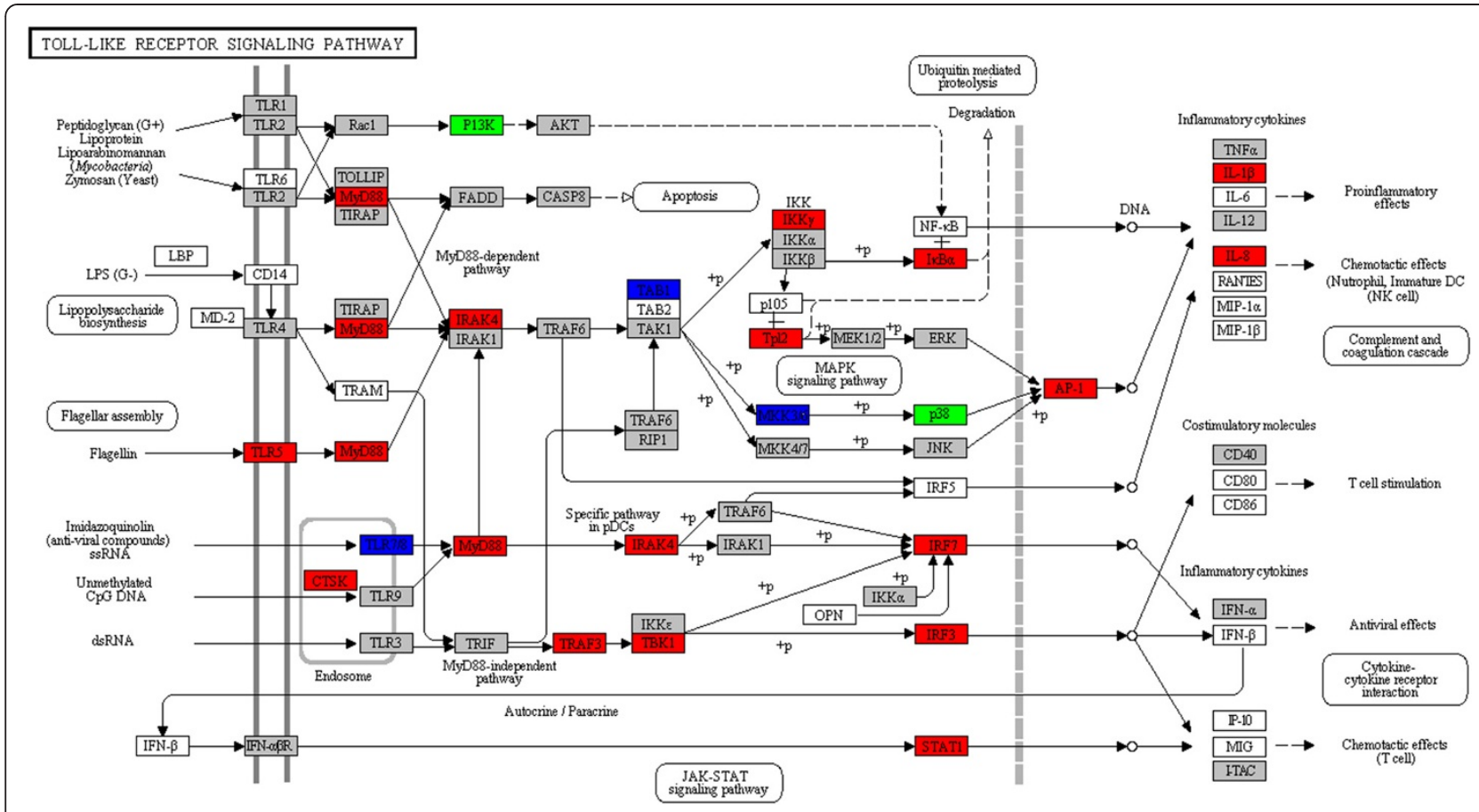

Figure 3 Significantly differentially expressed genes identified by KEGG as involved in toll-like receptor signaling. Red: significantly increased expression (fold-change $>2$ ); Blue: significantly decreased expression (fold-change <0.5); Green: genes detected both in up- and downregulated groups; Gray: unchanged expression.

regulated upon WED immunization, while 15 genes were strongly down-regulated (data not shown). In the antigen processing and presentation signaling pathway, most of the up-regulated genes were found to be interrelated with the MHC-I processing pathway, while most of the down-regulated genes were related to the MHC-II processing pathway. In mammals, antigen processing and presentation are essential for triggering the downstream cellular and/or humoral immune responses [41]. The KEGG results revealed that eight genes involved in the MHC-I pathway were up-regulated, and six genes involved in the MHC-II pathway were down-regulated by two days after WED immunization (Figure 4). These results suggested that the MHC-I related pathways were co-induced following WED immunization, while the MHC-II related pathways were co-depressed. This unique perspective should be further clarified.

\section{qPCR analysis of MHC processing pathways}

We next sought to further clarify the strength of the correlations of up-regulated genes to MHC-I processing pathway and down-regulated genes to MHC-II processing pathway in zebrafish during early stage following WED immunization. Differential expression of 12 genes associated with $\mathrm{MHC}$ antigen processing was analyzed by qPCR to confirm the hypothesis that antigen processing and presentation pathways elicit an adaptive immune response following immunization. The assay was performed with both spleen and liver samples collected over the first five days post immunization. Most of the results were consistent with those of the RNA-seq analysis (Figures 5 and 6). MHC-I processing pathway related gene expression in liver tissues from WED-immunized groups were significantly up-regulated relative to mockimmunized groups (Figure 5). The up-regulations of differentially expressed genes in liver mostly reappeared in spleen, except for hsp90a, hspa4a and calnexin. The down-regulation expression of the three genes in spleen might reflect their different functions in two immuneassociated organs. In contrast, the MHC-II processing pathway related gene expression was all down-regulated and completely coordinated in liver and spleen during the early stage following WED immunization (Figure 6). This showed that MHC-II processing pathway was inhibited in two immune organs by WED immunization. Thus, $\mathrm{CD} 4+\mathrm{T}$ cells activation could be depressed following immunization. The implications of this finding should be further investigated in teleost, specifically in zebrafish as model of E. tarda infection. In addition, the qPCR data also revealed that antigen processing in liver possesses a comparatively dominant role to that in spleen. The relatively intense expression in liver showed that antigen processing plays an essential role in WED-immunized zebrafish liver. 
Table 3 Differentially expressed genes related to the antigen processing and presentation pathways

\begin{tabular}{|c|c|c|c|c|c|c|}
\hline Gene name & Accession number & Description & RPKM-C & RPKM-V & Fold-change & $p$-value \\
\hline ciita & ENSDARG00000090851 & MHC class II, transactivator & 0.70 & 0.03 & -26.80 & $5.54 \mathrm{E}-07$ \\
\hline si:busm1-160c18.3 & ENSDARG00000093706 & Beta chain, MHC class II & 2.04 & 0.36 & -7.22 & 3.43E-04 \\
\hline$m h c 2 d a b$ & ENSDARG00000079105 & MHC class II DAB gene & 15.90 & 2.62 & -7.22 & 2.16E-17 \\
\hline si:busm1-194e12.12 & ENSDARG00000055447 & Novel MHC II beta chain protein & 6.12 & 1.70 & -4.33 & $1.70 \mathrm{E}-06$ \\
\hline lamp2 & ENSDARG00000014914 & Lysosomal membrane glycoprotein 2 & 3.47 & 1.11 & -4.14 & 5.36E-03 \\
\hline$c d 74$ & ENSDARG00000036628 & MHC, class II invariant chain, CD74 molecule, & 34.87 & 10.77 & -3.93 & $6.53 \mathrm{E}-12$ \\
\hline $\operatorname{ctsz}$ & ENSDARG00000043081 & Cathepsin Z & 4.67 & 1.51 & -3.77 & 8.36E-03 \\
\hline ctssb.1 & ENSDARG00000074656 & Cathepsin S, b.1 & 23.25 & 10.44 & -2.59 & 4.24E-06 \\
\hline$c d 9 a$ & ENSDARG00000005842 & CD9 antigen & 37.62 & 19.91 & -2.34 & $5.21 \mathrm{E}-05$ \\
\hline eif4a1b & ENSDARG00000003032 & CD68 antigen variant & 472.12 & 258.97 & -2.33 & $1.94 \mathrm{E}-05$ \\
\hline cd1511 & ENSDARG00000068629 & CD151 antigen, like & 0.71 & 4.88 & 4.80 & $1.16 \mathrm{E}-03$ \\
\hline psme3 & ENSDARG00000012234 & Proteasome activator complex subunit 3 & 8.68 & 19.69 & 2.02 & $6.74 \mathrm{E}-03$ \\
\hline$i t m 1$ & ENSDARG00000053832 & Integral membrane protein 1(STT3) & 121.55 & 311.95 & 2.03 & $6.23 \mathrm{E}-04$ \\
\hline psme2 & ENSDARG00000033144 & Proteasome activator PA28 subunit 2 & 10.22 & 25.23 & 2.04 & 1.27E-03 \\
\hline pomp & ENSDARG00000032296 & Proteasome maturation protein & 12.60 & 31.84 & 2.13 & 2.01E-03 \\
\hline tapbpl & ENSDARG00000058351 & TAP binding protein-like(TAPBPL) & 5.75 & 15.38 & 2.18 & $1.09 \mathrm{E}-03$ \\
\hline psmel & ENSDARG00000002165 & Proteasome activator PA28 subunit 1 & 12.99 & 39.58 & 2.65 & 4.72E-05 \\
\hline $\operatorname{can} x$ & ENSDARG00000037488 & Calnexin & 49.15 & 167.03 & 2.85 & $3.71 \mathrm{E}-06$ \\
\hline$c d 82 b$ & ENSDARG00000026070 & CD82 antigen,b & 5.33 & 19.59 & 2.99 & 5.56E-07 \\
\hline si:ch211-287j19.6 & ENSDARG00000001470 & MHC class I ZE protein & 4.96 & 18.39 & 3.09 & $1.63 \mathrm{E}-06$ \\
\hline$i t f g 1$ & ENSDARG00000075584 & T-cell immunomodulatory protein & 1.74 & 7.16 & 3.21 & $6.74 \mathrm{E}-07$ \\
\hline hspa4a & ENSDARG00000004754 & Heat shock protein $4 a$ & 5.42 & 18.17 & 3.28 & $9.21 \mathrm{E}-06$ \\
\hline psma5 & ENSDARG00000003526 & Proteasome (prosome, macropain) subunit, alpha & 1.90 & 7.52 & 3.60 & $9.48 \mathrm{E}-04$ \\
\hline calr & ENSDARG00000043276 & ER-resident chaperone calreticulin & 29.97 & 131.43 & 3.72 & 4.47E-10 \\
\hline psmb3 & ENSDARG00000013938 & Proteasome (prosome, macropain) subunit, beta & 13.34 & 60.15 & 3.80 & 3.27E-09 \\
\hline psmf1 & ENSDARG00000022652 & Proteasome (prosome, macropain) inhibitor & 3.38 & 15.05 & 3.94 & $1.51 \mathrm{E}-06$ \\
\hline psmg4 & ENSDARG00000090191 & Proteasome (prosome, macropain) & 2.39 & 11.31 & 4.22 & $6.25 \mathrm{E}-04$ \\
\hline hsp90a.2 & ENSDARG00000024746 & Heat shock protein HSP 90 kDa alpha 2 & 0.54 & 2.81 & 4.39 & 7.07E-05 \\
\hline psmcib & ENSDARG00000043561 & Proteasome (macropain) $26 \mathrm{~S}$ subunit & 12.62 & 67.60 & 4.55 & $2.86 \mathrm{E}-15$ \\
\hline hsp70 & ENSDARG00000021924 & Heat shock cognate $70 \mathrm{kDa}$ protein & 0.24 & 2.13 & 6.67 & $1.06 \mathrm{E}-05$ \\
\hline cd63 & ENSDARG00000025147 & CD63 antigen & 32.31 & 296.86 & 7.61 & 4.69E-20 \\
\hline$c d 276$ & ENSDARG00000003061 & CD276 molecule & 0.60 & 7.15 & 7.94 & 2.91E-08 \\
\hline cd97 & ENSDARG00000089904 & CD97 molecule & 1.40 & 13.32 & 8.03 & 1.35E-31 \\
\hline hsp90b1 & ENSDARG00000003570 & Endoplasmin (grp94) & 93.60 & 1118.80 & 10.06 & 2.85E-18 \\
\hline gata2a & ENSDARG00000059327 & GATA-binding protein $2 \mathrm{a}$ & 0.08 & 1.04 & 12.76 & 8.73E-03 \\
\hline$c t s / 1 a$ & ENSDARG00000007836 & Cathepsin L, 1 a & 20.92 & 340.42 & 13.53 & $1.98 \mathrm{E}-43$ \\
\hline dnajb11 & ENSDARG00000015088 & DnaJ (Hsp40) homolog, subfamily B & 3.42 & 61.99 & 15.13 & 1.19E-32 \\
\hline
\end{tabular}

Identification of all differentially expressed genes was based on $p<0.05$. A $p$-value $<0.05$ indicated that the gene was significantly altered in WED-immunized fish with respect to mock-immunized fish. The absolute value of "Fold-change" is the magnitude of up- or down-regulation for each gene/homolog after WED immunization. Fold-change $>2$ indicates up-regulation, and $<-2$ indicates down-regulation.

\section{Discussion}

At present, molecular studies on the immune response to pathogens in fish models are mainly focused on infectious disease pathogenesis. RNA-seq and microarraybased transcriptome profiling studies have revealed that the teleosts are useful in vivo models for identifying host determinants of responses to bacterial infection [27-30]. Furthermore, the RNA-seq approach has already been successfully applied to several infectious disease models of zebrafish [25-27]. However, none have applied the 


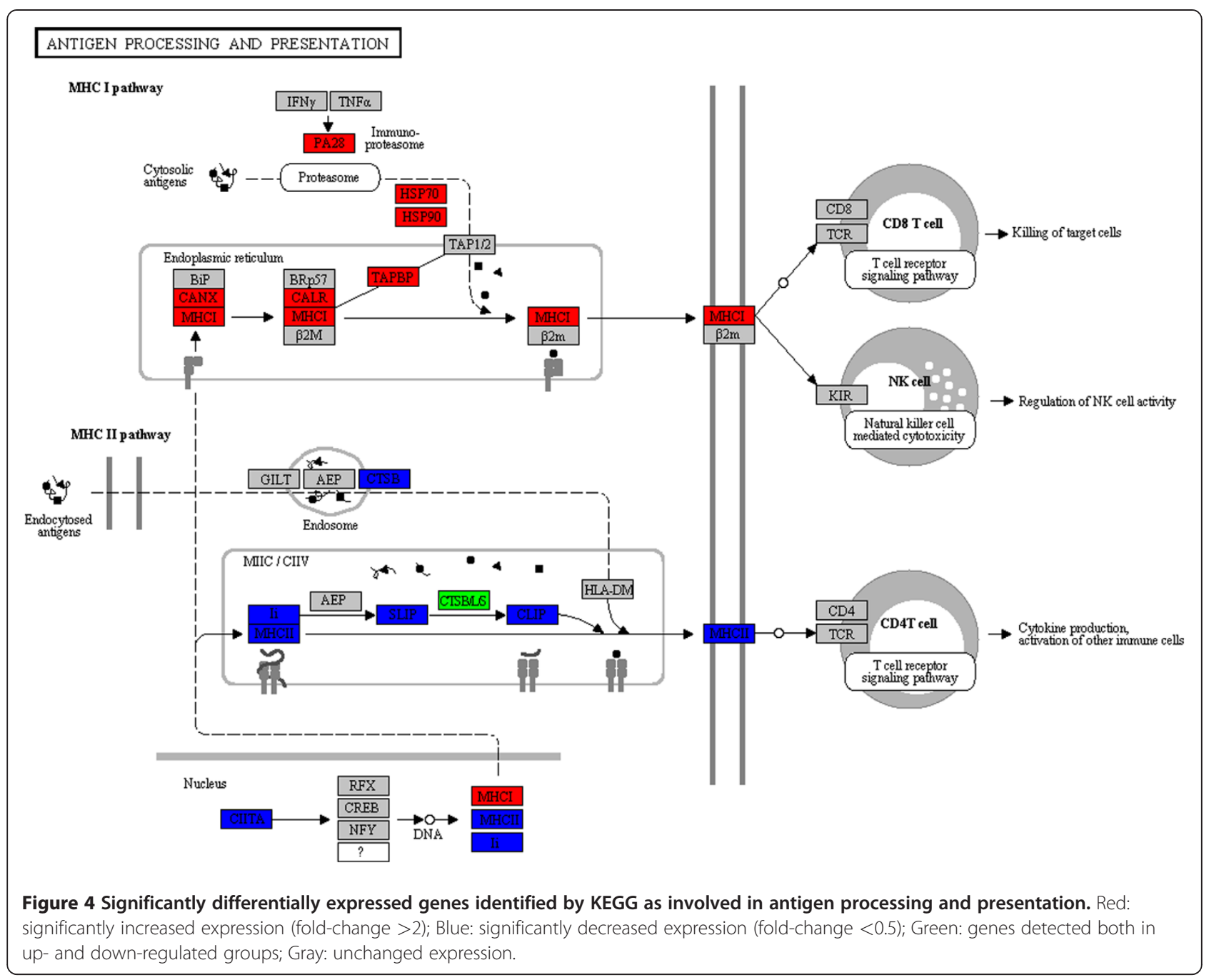

RNA-seq technology to elucidate the immune-related pathways underlying the zebrafish response to vaccination for more effective vaccine evaluation. In this work, in order to gain comprehensive insight into the immunogenetics of zebrafish following immunization with the putative E. tarda live attenuated vaccine, a high-throughput deep sequencing-by-synthesis technology was used to investigate the immunization-related gene expression patterns. DESeq analysis identified 4565 significantly differentially expressed genes in the zebrafish liver following WED immunization. GO and KEGG analysis revealed that the genes involved in the ER protein processing as well as the phagosome and antigen processing and presentation pathways are regulated at the early stage following WED immunization (Table 1 and Figure 2). Significantly, two class MHC pathways were found to be reversely regulated upon immunization, and the MHC class I pathway was activated and the MHC class II pathway was inhibited (Figures 4, 5 and 6). Both the RNAseq results and qPCR data from our study of zebrafish liver during the early stage after WED immunization indicated that activation of the MHC-I processing pathway in teleosts could elicit cellular immune responses for protection.

Once bacterial vaccines are administrated into the animal host, they are often internalized by phagocytes via different entry mechanism. However, the subsequent issues involved in microbial sensing and antigen processing are not well defined. In the conventional paradigm, MHC class II molecules present antigenic fragments acquired by the endocytic route to the immune system for recognition and activation of $\mathrm{CD} 4+\mathrm{T}$ cells [42]. MHC class I molecules, on the other hand, are restricted to surveying the cytosol for endogenous antigen from intracellular pathogens (such as bacteria, parasites, and viruses), tumors, or self-proteins, which are degraded into proteasomal products and then presented on MHC class I molecules to CD8+ T cells, thus exersting an irreplaceable role on cellular-mediated immuno-protection toward intracellular pathogens [43,44]. E. tarda is believed to be an intracellular pathogen that can survive 


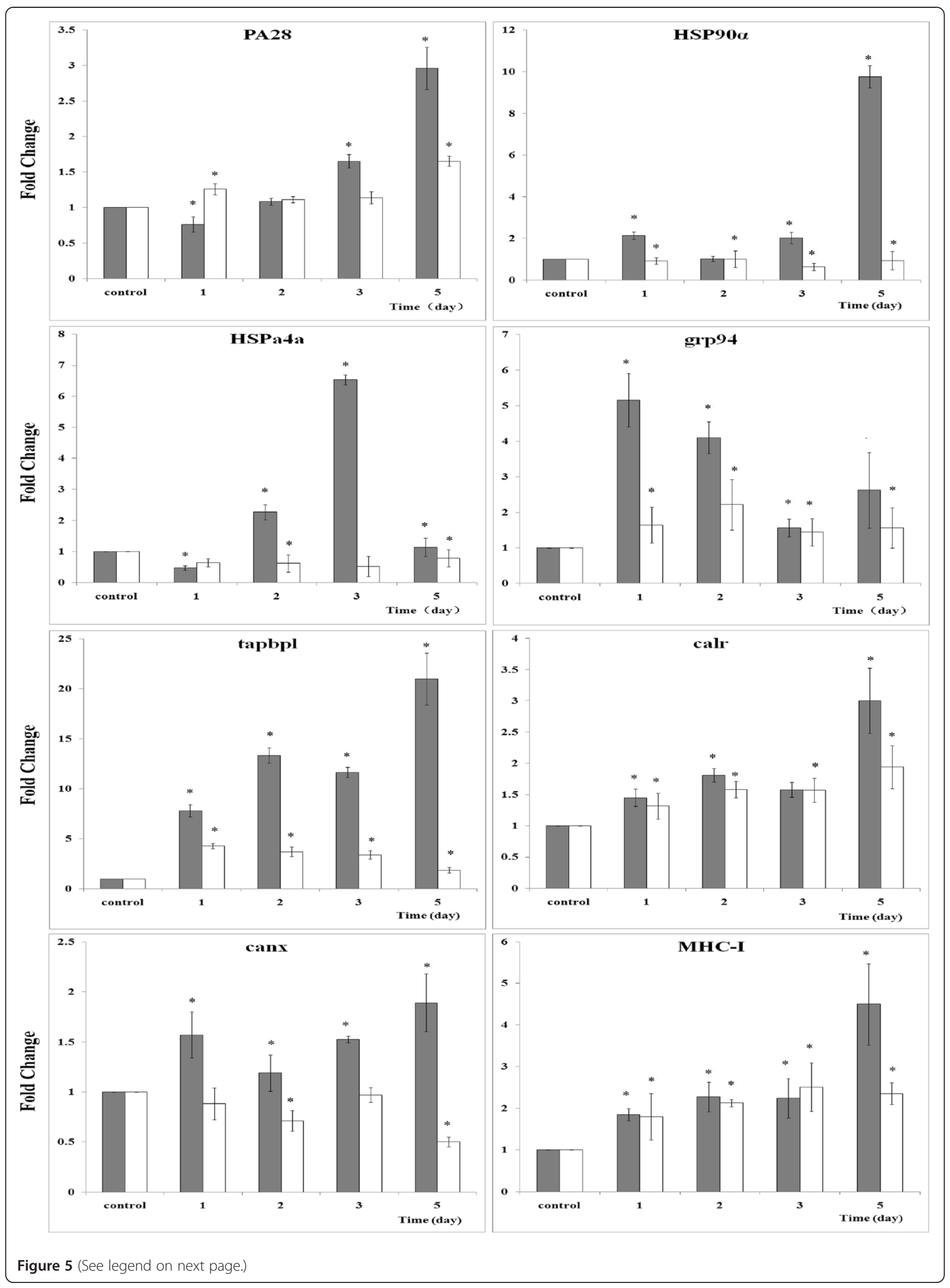


(See figure on previous page.)

Figure 5 qPCR analysis of the genes related to MHC-I processing pathway in zebrafish following WED-immunization during the first five days. Proteasome activator (PA28), heat shock protein 90 kDa alpha (HSP90a), heat shock protein 4a (HSPa4a), endoplasmin (grp94), TAP binding protein (tapbpl), calreticulin (calr), calnexin (canx) and MHC class I ZE protein (MHC-I) were validated. The relative expression of the above immune-related genes in livers (gray bars) and spleens (white bars) of zebrafish were analyzed by qPCR. Zebrafish were vaccinated via intramuscular injection with WED or PBS. The livers and spleens of 10 fish were taken at 1, 2, 3, 5 d post-vaccination, respectively, and total RNA was extracted and used for qPCR. The mRNA level of each immune-related gene was normalized to that of $\beta$-actin. For the gene of each time point, values represent fold change in expression compared to the control treatment, which was set at 1.0. Results are expressed as means \pm SD $(n=3)$. Mock immunized group was subtracted from each group. Independent-sample t-test in the SPSS software (Version 11.5, SPSS Inc.) was used to determine statistical significance of the WED immunized groups relative to mock groups. Significant differences were considered at ${ }^{*} p<0.01$.

and replicate within large phagosomes in macrophages [45]. Since WED is an attenuated strain from wild type E. tarda, it could be assumed that WED bacteria possess the ability to survive in phagosomes of APC cells and the internalized bacteria are recognized as endogenous or exogenous antigen which would be presented or cross-presented by the MHC-I pathway, and finally evoking a CD8+ CTL-mediated response to achieve immune protection.

In MHC-I antigen processing pathway, antigenic peptides are degraded in the cytoplasm by proteasome, then translocated into the ER and loaded onto MHC-I molecules with the help of several protein components. PA28, as an important proteasome activator, is a heterohexameric ring that binds to one or both ends of the $20 \mathrm{~S}$ proteasome $[43,46]$. Upon binding, it increases the catalytic activity of all three of the proteasome active sites, leads to changes in substrate cleavage, thereby generating more MHC class I-presented peptides $[46,47]$. Khan et al. [48] reported that constitutive proteasomes were replaced with immune-proteasomes in mice livers starting at two days after Listeria monocytogenes infection. Immuno-proteasomes support the generation of MHC class I epitopes and shape immune-dominance hierarchies of CD8+ T cells [46]. In mice, this switch is marked by the up-regulation of proteasome activator PA28 subunits, which alter the fragmentation of polypeptides through the proteasome and are inducible by
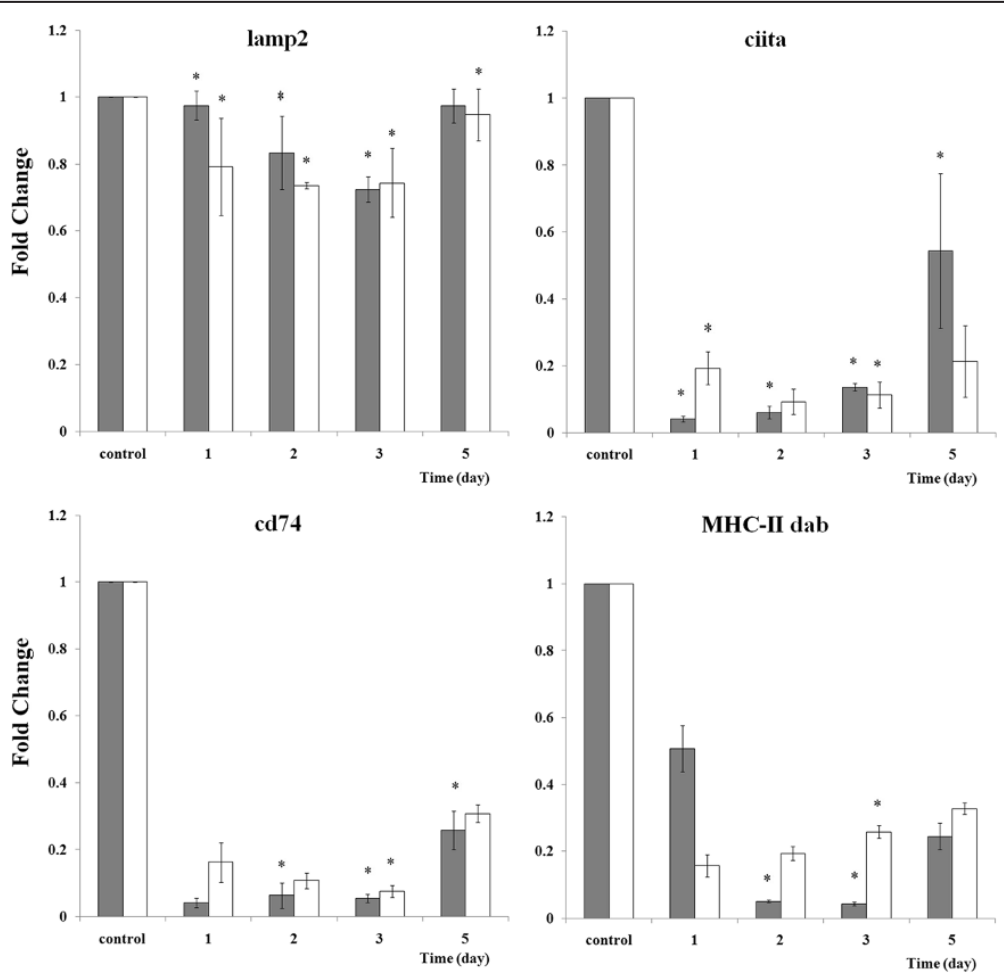

Figure 6 qPCR analysis of the genes related to MHC-Il processing pathway in zebrafish following WED-immunization during the first five days. Lysosomal membrane glycoprotein 2 (lamp2), MHC class II DAB gene (MHC-II dab), CD74 molecule (cd74) and MHC class II, transactivator (CIITA) were validated. GPCR analysis for the expressions of the above immune-related genes in livers (gray bars) and spleens (white bars) of zebrafish vaccinated with WED. The experimental procedure was the same as in the legend of Figure 5. 
IFN- $\gamma$ [48]. The study of immune responses to E. ictaluri infection in blue catfish liver demonstrated that both the PA28 $\alpha$ and PA28 $\beta$ were up-regulated [34]. In the study described herein, the genes encoding PA28 subunit 1, PA28 subunit 2 and PA28 subunit 3 were all upregulated in zebrafish liver, which suggested a shift toward MHC class I antigen processing occurred at the early stage after WED immunization.

Heat shock proteins (HSP) are a type of highly conserved and ubiquitously expressed proteins that play an essential role as molecular chaperones in protein folding and transport within the cell [49] and possess the ability to stimulate MHC class I antigen processing [50]. HSP/ peptide complexes are taken up by APC via specific receptors, whose signaling leads to MHC-I presentation of HSP-associated peptides and the induction of specific CD8+ cytotoxic T cells [50]. The antigenic peptides chaperoned by HSPs are known to be more efficient, by orders of magnitude, than the free peptides for presentation by MHC-I $[49,50]$. In our work, three heat shock proteins (heat shock cognate $70 \mathrm{kDa}$ protein, heat shock protein $4 \mathrm{a}$ and heat shock protein $90 \mathrm{kDa}$ alpha 2) were found to be up-regulated following WED immunization, and the activated HSPs suggested that the internalized WED bacteria were processed and loaded onto MHC class I molecules, ultimately initiating initiate the CTLs.

As cited above, MHC class I molecules present antigenic peptides on cell surface for recognition by CD8+ T cells [43]. Like other glycoproteins, the folding and assembly of MHC class I molecules require interactions with a number of chaperone molecules in the ER, some of which are specific to MHC class I molecules [44]. Among the known ER chaperones, endoplasmin (grp94) possesses the ability to bind peptides suitable for assembly on to MHC class I molecules together with calreticulin [51]. Calreticulin and calnexin are specialized ER lectin-binding chaperones to bind transiently to newly-synthesized glycoproteins, but the calreticulin has been suggested as unique to interactions with the HSP/grp94 complex, which leads to recruitment of ER protein 57 [52]. The interaction between calnexin and MHC class I molecules is believed to stabilize the class I heavy chain and help it to associate with the $\beta 2 \mathrm{~m}$ component $[51,53]$. In this work, the three ER chaperons, calreticulin, calnexin and endoplasmin (grp94), were all found to be induced in WED-immunized zebrafish liver, providing further evidence that an active MHC class I processing pathway was stimulated by WED immunization. In addition, TAP binding protein, another molecule involved in MHC class I antigen loading [44,49,51,53], and MHC class I complex ZE protein were also up-regulated in WED-immunized zebrafish liver, strongly suggesting a vigorous activation of the MHC-I processing pathway.

The MHC antigen processing-associated genes from zebrafish have been extensively characterized. However, little is known about their expression patterns in zebrafish following vaccine immunization. Recently, the coordinated up-regulation of MHC class I-related components including MHC class I alpha chain, $\beta 2 \mathrm{~m}$, calreticulin, endoplasmin, PA28 $\alpha$ and PA28 $\beta$ were reported in large yellow croaker following poly I:C injection [54] and in catfish following an intracellular bacterial infection [34]. In this work, the RNA-seq data were given to show a coordinated down-regulation of several MHC class II antigen processing and presentation components, including the MHC-II DAB, MHC-II beta chain, MHC-II invariant chain (CD74), MHC class II transactivator (CIITA), cathepsin B and lysosomal membrane glycoprotein 2 (lamp2). This complex process is illustrated in Figure 4 and the differentially expressed genes are listed in Table 3. Furthermore, qPCR data confirmed the co-inhibition of lamp2, MHC-II dab, CD74, and CIITA in zebrafish liver and spleen (Figure 6). In previous researches, a remarkable inhibition of MHC-II expression and antigen presentation was ever reported in some pathogen infection models, including Brucella abortus [55], and Mycobacterium tuberculosis [56-58]. For pathogens, an ability to impair the antigen processing and presentation of host has been proposed to facilitate chronic infection by decreasing $\mathrm{T}$ cell responses to microbial antigens. For vaccines, however, the underlying significance of suppression of the MHC-II expression and antigen presentation remains unknown.

\section{Conclusions}

In conclusion, in this work, zebrafish was used as a model to investigate the host immune mechanisms underlying the protective effects of the E. tarda live attenuated vaccine. RNA-seq data revealed that the coordinate up-regulation of MHC-I processing pathways and down-regulation of MHC-II-associated pathways occurred at the early stage of vaccine immunization, providing insights into the molecular mechanisms of immune protection. The successful application of RNAseq technology in the vaccine-zebrafish interaction model in this work established a new experimental platform for investigating the vaccine-specific host immune responses in a comprehensive and sensitive manner. Future studies using this approach will likely provide further significant insights into the detailed mechanisms of teleost immunity that will benefit the aquaculture industry, both from economic and human food source perspectives.

\section{Methods}

\section{Fish and immunization}

Healthy zebrafish (Danio rerio), weighing $0.3 \pm 0.1 \mathrm{~g}$ and about 6 months of age, were obtained from the animal center at the East China University of Science and 
Technology (Shanghai, China) and maintained at $22 \pm 2^{\circ}$ $\mathrm{C}$ in a zebrafish cultivation system with a photo-period of 12:12 h (light : dark). Aquaria were supplied with flow-through dechlorinated and continuously aerated water at a rate of approximately $2 \times 10^{-4} \mathrm{~min}^{-1}$. After at least one week of acclimatization, they were randomly divided into six treatment groups (70 fish per group) including three immunized groups (V1-V3) and three control groups (C1-C3), and the fish in each group were cultured in a separate tank. The fish in V1-V3 groups were intramuscularly (i.m.) injected with $1 \times 10^{5}$ CFU.fish $^{-1}$ of WED bacteria in $5 \mu$ phosphate buffered saline (PBS), as previously described [12], and the fish in C1C3 groups were i.m. injected with $5 \mu \mathrm{l}$ PBS alone. After two days of immunization, 20 fish from each of the three WED-immunized and three mock-immunized groups were sacrificed under anesthesia to obtain liver samples, and subsequently stored at $-80^{\circ} \mathrm{C}$ until RNA extraction for RNA-seq analysis. Meanwhile, 10 fish from each group were sacrificed under anesthesia at days 1, 2, 3, and 5 post-immunization to obtain liver and spleen tissue samples, and subsequently stored at $-80^{\circ} \mathrm{C}$ until RNA extraction for real-time qPCR analysis. All the zebrafish were handled in compliance with the local animal welfare regulations and maintained according to standard protocols (http://ZFIN.org). The immunization experiment was approved by the animal center at the East China University of Science and Technology (Shanghai, China).

\section{Library preparation and sequencing}

Total RNA was extracted from each tissue sample using the TRIzol reagent (Invitrogen, USA) according to the manufacturer's instructions. To remove residual genomic DNA, the RNA samples were incubated with 10 units of DNA-free DNAse I (Ambion, USA) for $30 \mathrm{~min}$ at $37^{\circ} \mathrm{C}$. The quality and quantity of the purified RNA were determined by measuring the absorbance at $260 \mathrm{~nm} / 280 \mathrm{~nm}\left(\mathrm{~A}_{260} / \mathrm{A}_{280}\right)$ using a Nanodrop ND-1000 spectrophotometer (LabTech, USA). RNA integrity was further verified by electrophoresis through a $1.5 \%(\mathrm{w} / \mathrm{v})$ agarose gel.

Poly (A) mRNA was isolated from the total RNA samples with oligo (dT) magnetic beads (Invitrogen). The purified mRNA was fragmented by the RNA fragmentation kit (Ambion) and applied as template for firststrand cDNA synthesis using random hexamer primers and reverse transcriptase (Invitrogen). The secondstrand cDNA was synthesized using RNase H (Invitrogen) and DNA polymerase I (New England Biolabs, USA). The Illumina Genomic DNA Sample Prep kit (Illumina, USA) was used to generate $120 \mathrm{bp}$ paired-end (PE) cDNA libraries by following the manufacturer's protocol. The libraries were loaded onto flow cell channels for sequencing on the Illumina HiSeq 2000 instrument by the Chinese National Human Genome Center (Shanghai, China). A total of six paired-end cDNA libraries of zebrafish livers were constructed for each of the test groups of WED-immunized and mockimmunized fish. Triplicate biological replicates were performed for each group. Raw data (tag sequences) were deposited in the NCBI database under submission number SRA048658.2.

\section{Transcriptome analysis}

The Illumina HiSeq 2000 system-generated 120 bp raw PE reads were first processed by the FASTX-Toolkit to remove the reads with sequencing adaptors and of low quality (phred quality <5). Then, the Burrows-Wheeler Aligner's Smith-Waterman Alignment (BWA) program was used to align the remaining reads to the reference zebrafish mRNA from the Ensembl database [59]. The transcription level of each gene was deduced by determining the total number of reads mapped to each gene using Picard tools (http://picard.sourceforge.net/). Differentially expressed genes were identified by the DESeq package in $\mathrm{R}$ software [60], using two-fold change ( $\log _{2}$ (fold-change) $\geq 1$ or $\leq-1$ ) and $p$-value $<0.05$ (cut-off at $5 \%$ false discovery rate (FDR)) as the threshold. After data normalization by the $p$-value and FDR calculation, the resulting expression intensity values were analyzed by the MA plot-based method, as described by Wang et al. [32].

\section{Functional analysis of differentially expressed genes}

The Database for Annotation, Visualization and Integrated Discovery (DAVID, v6.7) [61] was used to investigate functional enrichment for over- and underexpressed genes by more than two-fold in the WEDimmunized group relative to the mock-immunized group. Gene functional enrichment was performed using the default parameters in DAVID to obtain an adjusted $p$-value $<0.05$ for the test gene group versus the zebrafish gene ontology (GO) annotation set. The foldenrichment cut-off suggested for DAVID functional annotation is 1.5 [61]. In addition, the significantly up-regulated genes from the differentially expressed genes dataset were further analyzed by investigating the corresponding GO biological processes. Furthermore, GO analysis of genes transcribed at different levels was also performed using the Biological Networks Gene Ontology (BiNGO) tool, which is based on the Cytoscape software (www.cytoscape.org). The hypergeometric test with Benjamini \& Hochberg False Discovery Rate (FDR) was performed using the default parameters to obtain an adjusted $p$-value $(<0.05)$ between the test gene group and the merged non-redundancy zebrafish (Danio rerio) and mouse (Mus musculus) GO annotation set. Finally, 
the web-based Kyoto Encyclopedia of Genes and Genomes (KEGG) pathway analysis program run by the KEGG Automatic Annotation Server (KAAS) (http:// www.genome.jp/tools/kaas/) was used to obtain functional annotation of genes by performing basic local alignment search tool (BLAST)-mediated comparisons against the manually-curated KEGG GENES database [62]. We merged the most current KEGG GENES entries for Danio rerio and Mus musculus to generate a reference dataset and used the bi-directional best hit (BBH) information method to further analyze the significantly differentially expressed genes to gain insights into the related biological pathways.

\section{qPCR analysis}

To verify the differential expression detected by sequencing, qPCR was performed using the ABI Prism 7500 Detection System (Applied Biosystems, USA) with SYBR Green (Roche, USA) as the fluorescent detection dye, according to the manufacturer's protocol. First-strand cDNA was synthesized from $1 \mu \mathrm{g}$ of total mRNA, as described above, and applied as a template for qPCR with gene-specific primers (Additional file 4). Primers were designed using Primer Express 3 software. To determine the PCR efficiency, we first generated a standard curve by amplifying ten-fold serial dilutions of cDNA using primers to both the gene of interest and an internal control $(\beta$-actin), and all primers were optimized until PCR efficiency values fell in 1.80-2.15. The qPCR thermal cycling conditions for all reactions were $95^{\circ} \mathrm{C}$ for $15 \mathrm{~min}$, followed by 40 cycles of $95^{\circ} \mathrm{C}$ for $5 \mathrm{~s}, 60^{\circ} \mathrm{C}$ for $20 \mathrm{~s}$, and $72^{\circ} \mathrm{C}$ for $20 \mathrm{~s}$. All qPCR reactions were performed for three biological replicates, and the data for each sample were expressed relative to the expression levels of $\beta$-actin by using the $2^{-\Delta \Delta C T}$ method [63]. Independent-sample $t$-test in the SPSS software (Version 11.5, SPSS Inc.) was used to determine statistical significance. Significant differences were considered at $p<0.01$.

\section{Additional files}

Additional file 1: Solexa libraries of the WED immunized and PBS mock zebrafish liver. Six Solexa cDNA libraries were constructed from the livers of mock-immunized and WED-immunized zebrafish. Biological replicates (C1-C3 and V1-V3) were pooled to make representative samples for deep sequencing analysis. To assess the quality of sequencing, the reads were mapped to the zebrafish reference genome with no more than 5-mismatches. The sequencing data for $\mathrm{C} 1$-read 1 to C3-read 2 and V1-read 1 to V3-read 2 corresponded to the paired end sequencing (forward and reverse sequencing) data in each library, respectively.

\section{Additional file 2: Annotation results of $\mathbf{4 5 6 5}$ differentially} expressed genes. Transcription level of each gene was deduced by determining the total number of reads mapped to each gene using Picard tools. Differentially expressed genes were identified by the DESeq package in $R$ software using two-fold change ( $\log _{2}$ (fold-change) $\geq 1$ or $\leq-1$ ) and $p$-value $<0.05$ (cut-off at $5 \%$ false discovery rate (FDR)) as the threshold. "baseMean", the base mean of counts divided by the size factors. "baseMean A", the base mean for the counts of PBS-mock condition. "baseMean B", the base mean for the counts of WEDimmunized condition. "rpkm_avg_c", the expression level of PBS-mock group. "rpkm_avg_v", the expression level of WED-immunized group. "ZF-Annotation", annotation of the differentially expressed genes through BLASTN similarity searches against the Ensembl zebrafish RefSeq mRNA database.

Additional file 3: GO function annotation results of $\mathbf{4 5 6 5}$ differentially expressed genes. Functional enrichment for over- and under-expressed genes by more than two-fold in the WED-immunized group relative to the mock-immunized group generated by DAVID (v6.7). Gene functional enrichment was performed using the default parameters in DAVID to obtain an adjusted $p$-value $<0.05$ for the test gene group versus the zebrafish gene ontology (GO) annotation set. The foldenrichment cut-off suggested by DAVID functional annotation was 1.5. The Biological Progress (BP-up and BP-down), Cellular Components (CC-up and CC-down), and Molecular Functions (MF-up and MF-down) associated with the $\mathrm{GO}$ analysis at different levels were also analyzed by BiNGO software. The hypergeometric test with Benjamini \& Hochberg FDR were performed by the default parameters to obtain an adjusted $p$-value $(<0.05)$ between the test gene group and the merged non-redundancy zebrafish (Danio rerio) and mouse (Mus musculus) GO annotation set

Additional file 4: Primers for quantitative real-time PCR.

\section{Competing interests}

The authors declare that there are no competing interests.

\section{Acknowledgements}

This work was supported by grants from the National High Technology Research and Development Program of China (No. 2008AA092501), the earmarked fund for China Agro-industry Research System (No. CARS-50), and the Science Foundation of Ministry of Education of China (No. Ncet-09-0344).

\section{Authors' contributions}

DY and QL carried out the bioinformatics analyses and wrote the manuscript. MY participated in the bioinformatics analysis. DY performed the laboratory experiments. HW, QW, JX and YZ conceived the study and helped write the manuscript. All authors read and approved the final manuscript.

Received: 10 February 2012 Accepted: 17 July 2012

Published: 17 July 2012

\section{References}

1. Felix E, Graham JL: Zebrafish as a model for vertebrate hematopoiesis. Curr Opin Pharmacol 2010, 10:563-570.

2. Lieschke GJ, Currie PD: Animal models of human disease: zebrafish swim into view. Nat Rev Genet 2007, 8:353-367.

3. Meeker N, Trede N: Immunology and zebrafish: spawning new models of human disease. Dev Comp Immunol 2008, 32:745-757

4. Sullivan C, Kim CH: Zebrafish as a model for infectious disease and immune function. Fish Shellfish Immunol 2008, 25:341-350.

5. Chao CC, Hsu PC, Jen CF, Chen IH, Wang CH, Chan HC, Tsai PW, Tung KC, Wang $C H$, Lan CY, Chuang YJ: Zebrafish as a model host for candida albicans infection. Infect Immun 2010, 78:2512-2521.

6. Vojtech LN, George ES, Carla C, Ostland V, Hansen JD: Host immune response and acute disease in a zebrafish model of francisella pathogenesis. Infect Immun 2009, 77:914-925.

7. Van der Sar AM, Appelmelk BJ, Vandenbroucke-Grauls CMJE, Bitter W: A star with stripes: zebrafish as an infection model. Trends Microbiol 2004, 12:451-457

8. Kanther M, Rawls JF: Host-microbe interactions in the developing zebrafish. Curr Opin Immunol 2010, 22:10-19.

9. Tobin DM, May RC, Wheeler RT: Zebrafish: A see-through host and a fluorescent toolbox to probe host-pathogen interaction. PLOS Pathog 2012, 8(1):e1002349.

10. Novoa B, Romero A, Mulero V, Rodríguez I, Fernández I, Figueras A: Zebrafish (Danio rerio) as a model for the study of vaccination against viral haemorrhagic septicemia virus (VHSV). Vaccine 2006, 24:5806-5816. 
11. Cui Z, Samuel-Shaker D, Watral V, Kent ML: Attenuated mycobacterium marinum protects zebrafish against mycobacteriosis. J Fish Dis 2010, 33:371-375

12. Xiao JF, Chen T, Wang QY, Liu Q, Wang X, Lv YZ, Wu HZ, Zhang YX: Search for live attenuated vaccine candidate against edwardsiellosis by mutating virulence-related genes of fish pathogen Edwardsiella tarda. Lett Appl Microbiol 2011, 53:430-437.

13. Xu XP, Zhang LC, Weng SP, Huang ZJ, Lu J, Lan DM, Zhong XJ, Yu XQ, Xu AL, He JG: A zebrafish (Danio rerio) model of infectious spleen and kidney necrosis virus (ISKNV) infection. Virology 2008, 376:1-12.

14. Crispe IN: The liver as a lymphoid organ. Annu Rev Immunol 2009, 27:147-163.

15. Hofmann J, Greter M, Du Pasquier L, Becher B: B-cells need a proper house, whereas T-cells are happy in a cave: the dependence of lymphocytes on secondary lymphoid tissues during evolution. Trends Immunol 2010, 31:144-153.

16. Dancygier H: The liver as an immune organ. Clin Hepatol 2010, 11:141-152.

17. Wittamer V, Bertrand JY, Gutschow PW, Traver D: Characterization of the mononuclear phagocyte system in zebrafish. Blood 2011, 117:7126-7135.

18. Plitas G, Burt BM, Stableford JA, Nguyen HM, Welles AP, DeMatteo RP: Dendritic cells are required for effective cross-presentation in the murine liver. Hepatology 2008, 47:1343-1351.

19. Warren A, Le Couteur DG, Fraser R, Bowen DG, McCaughan GW, Bertolino P: T lymphocytes interact with hepatocytes through fenestrations in murine liver sinusoidal endothelial cells. Hepatology 2006, 44:1182-1190.

20. Ebrahimkhani MR, Mohar I, Crispe IN: Cross-presentation of antigen by diverse subsets of liver cells. Hepatology 2011, 54:1379-1387.

21. Gao B, Jeong W, Tian ZG: Liver: an organ with predominant innate immunity. Hepatology 2008, 47:729-736.

22. Morozova O, Hirst M, Marra MA: Applications of new sequencing technologies for transcriptome analysis. Annu Rev Genomics Hum Genet 2009, 10:135-151.

23. Han X, Wu X, Chung WY, Li T, Nekrutenko A, Altman NS, Chen G, Ma H: Transcriptome of embryonic and neonatal mouse cortex by highthroughput RNA sequencing. Proc Natl Acad Sci USA 2009, 106:12741-12746.

24. Tang F, Barbacioru C, Wang Y, Nordman E, Lee C, Xu N, Wang X, Bodeau J, Tuch BB, Siddiqui A, Lao K, Surani MA: mRNA-Seq whole-transcriptome analysis of a single cell. Nat Methods 2009, 6:377-382

25. Hegedus Z, Zakrzewskaa A, Ágostonb VC, Ordas A, Rácz P, Mink M, Spaink $H P$, Meijer AH: Deep sequencing of the zebrafish transcriptome response to mycobacterium infection. Mol Immunol 2009, 46:2918-2930.

26. Ordas A, Hegedus Z, Henkel CV, Stockhammer OW, Butler D, Jansen HJ, Racz P, Mink M, Spaink HP, Meijer AH: Deep sequencing of the innate immune transcriptomic response of zebrafish embryos to Salmonella infection. Fish Shellfish Immunol 2011, 31:716-724.

27. Liselotte $V$, Hong J, Per U, Outi H, Juha K: The zebrafish transcriptome during early development. BMC Dev Biol 2011, 11:30.

28. Van der Sar AH, Spaink HP, Zakrzewska A, Bitter W, Meijer AH: Specificity of the zebrafish host transcriptome response to acute and chronic mycobacterial infection and the role of innate and adaptive immune components. Mol Immunol 2009, 46:2\317-2332.

29. Mu YN, Ding F, Cui P, Ao JG, Hu SN, Chen XH: Transcriptome and expression profiling analysis revealed changes of multiple signaling pathways involved in immunity in the large yellow croaker during Aeromonas hydrophila infection. BMC Genomics 2010, 11:506.

30. Xiang LX, He D, Dong WR, Zhang YW, Shao JZ: Deep sequencing-based transcriptome profiling analysis of bacteria-challenged Lateolabrax japonicus reveals insight into the immune-relevant genes in marine fish. BMC Genomics 2010, 11:472.

31. Mohanty BR, Sahoo PK: Edwardsiellosis in fish: a brief review. J Biosci 2007, 32:1331-1344

32. Wang $L K$, Feng $Z X$, Wang $X$, Wang $X W$, Zhang $X G$ : DEGseq: an $R$ package for identifying differentially expressed genes from RNA-seq data. Bioinformatics 2010, 26:136-138.

33. Gerwick L, Corley-Smith G, Bayne CJ: Gene transcript changes in individual rainbow trout livers following an inflammatory stimulus. Fish Shellfish Immunol 2007, 22:157-171.

34. Peatman E, Terhune J, Baoprasertkul P, Xu P, Nandi S, Wang S, Somridhivej B, Kucuktas H, Li P, Dunham R, Liu Z: Microarray analysis of gene expression in the blue catfish liver reveals early activation of the MHC class I pathway after infection with Edwardsiella ictaluri. Mol Immunol 2008, 45:553-566.

35. Lin B, Chen SW, Cao Z, Lin YQ, Mo DZ, Zhang HB, Gu JD, Dong ML, Liu ZH, Xu AL: Acute phase response in zebrafish upon Aeromonas salmonicida and Staphylococcus aureus infection: Striking similarities and obvious differences with mammals. Mol Immunol 2007, 44:295-301.

36. Nakao M, Tsujikura M, Ichiki S, Vo TK, Somamoto T: The complement system in teleost fish: progress of post-homolog-hunting researches. Dev Comp Immunol 2011, 35:1296-1308.

37. John BV, Sharifa L: The binding and transport of alternative metals by transferring. Biochimica et Biophysica Acta 2012, 1820:362-378.

38. Stockhammer OW, Zakrzewska A, Hegedu s Z, Spaink HP, Meijer AH: Transcriptome profiling and functional analyses of the zebrafish embryonic innate immune response to Salmonella infection. J Immunol 2009, 182:5641-5653.

39. Sepulcre MP, Francisca AP, Azucena LM, Roca FJ, Meseguer J, Cayuela ML, Mulero $V$ : Evolution of lipopolysaccharide (LPS) recognition and signaling: fish TLR4 does not recognize LPS and negatively regulates NFKB activation. J Immunol 2009, 182:1836-1845

40. Seki E, Brenner DA: Toll-like receptors and adaptor molecules in liver disease: update. Hepatology 2008, 48:322-335.

41. Vyas JM, Van der Veen AG, Ploegh HL: The known unknowns of antigen processing and presentation. Nat Rev Immunol 2008, 8:607-618.

42. Van den Hoorn T, Paul P, Jongsma ML, Neefjes J: Routes to manipulate MHC class II antigen presentation. Curr Opin Immunol 2011, 23:88-95.

43. Sijts EJ, Kloetzel PM: The role of the proteasome in the generation of MHC class I ligands and immune responses. Cell Mol Life Sci 2011 68:1491-1502

44. Ramachandra L, Simmons D, Harding CV: MHC molecules and microbial antigen processing in phagosomes. Curr Opin Immunol 2009, 21:98-104.

45. Okuda J, Arikawa Y, Takeuchi Y: Intracellular replication of Edwardsiella tarda in murine macrophage is dependent on the type III secretion system and induces an up-regulation of anti-apoptotic NF-kB target genes protecting the macrophage from staurosporine-induced apoptosis. Microb Pathog 2006, 41:226-240.

46. Rock KL, Farfán-Arribas DJ, Shen L: Proteases in MHC class I presentation and cross-presentation. J Immunol 2010, 184:9-15.

47. Yamano T, Sugahara H, Mizukami S, Murata S, Chiba T, Tanaka K, Yui K, Udono H: Allele-selective effect of PA28 in MHC class I antigen processing. J Immunol 2008, 181:1655-1664.

48. Khan S, van den Broek M, Schwarz K, de Giuli R, Diener PA, Groettrup M: Immunoproteasomes largely replace constitutive proteasomes during an antiviral and antibacterial immune response in the liver. J Immunol 2001, 167:6859-6868.

49. Hartl FU: Molecular chaperones in cellular protein folding. Nature 1996 381:571-579.

50. Osterloh A, Breloer M: Heat shock proteins: linking danger and pathogen recognition. Med Microbiol Immunol 2008, 197:1-8.

51. Nicchitta CV, Reed RC: The immunological properties of endoplasmic reticulum chaperones: a conflict of interest? Essays Biochem 2000, 36:15-25.

52. Ireland BS, Brockmeier U, Howe CM, Elliott T, Williams DB: Lectin-deficient calreticulin retains full functionality as a chaperone for class I histocompatibility molecules. Mol Biol Cell 2008, 19:2413-2423.

53. Crotzer VL, Blum JS: Autophagy and its role in MHC-mediated antigen presentation. J Immunol 2009, 182:3335-3341

54. Liu G, Zheng W, Chen X: Molecular cloning of proteasome activator PA28-beta subunit of large yellow croaker (Pseudosciana crocea) and its coordinated up-regulation with MHC class I alpha-chain and beta(2)microglobulin in poly I:C-treated fish. Mol Immunol 2007, 44:1190-1197.

55. Barrionuevo P, Cassataro J, Delpino MV: Brucella abortus inhibits major histocompatibility complex class II expression and antigen processing through interleukin-6 secretion via toll-like receptor 2. Infect Immun 2008, 76:250-262.

56. Torres M, Ramachandra L, Rojas RE, Bobadilla K, Thomas J, Canaday DH, Harding CV, Boom WH: Role of phagosomes and major histocompatibility complex class II (MHC-II) compartment in MHC-II antigen processing of Mycobacterium tuberculosis in human macrophages. Infect Immun 2006, 74:1621-1630

57. Reuter MA, Pecora ND, Harding CV, Canaday DH, McDonald D: Mycobacterium tuberculosis promotes HIV trans-infection and suppresses 
major histocompatibility complex class II antigen processing by dendritic cells. J Virol 2010, 84:8549-8560.

58. Noss EH, Harding CV, Boom WH: Mycobacterium tuberculosis inhibits MHC Class II antigen processing in murine bone marrow macrophages. Cell Immunol 2000, 201:63-74.

59. Li H, Durbin R: Fast and accurate long-read alignment with BurrowsWheeler transform. Bioinformatics 2010, 26:589-595.

60. Anders S, Huber W: Differential expression analysis for sequence count data. Genome Biol 2010, 11:R106.

61. Huang DW, Sherman BT, Lempicki RA: Systematic and integrative analysis of large gene lists using DAVID bioinformatics resources. Nat Protocols 2009, 4:44-57.

62. Moriya Y, Itoh M, Okuda S, Yoshizawa AC, Kanehisa M: KAAS: an automatic genome annotation and pathway reconstruction server. Nucl Acids Res 2007, 35:182-185.

63. Thomas DS, Livak K: Analyzing real-time PCR data by the comparative CT method. Nat Protocols 2008, 3:1101-1108.

doi:10.1186/1471-2164-13-319

Cite this article as: Yang et al:: RNA-seq liver transcriptome analysis reveals an activated MHC-I pathway and an inhibited MHC-II pathway at the early stage of vaccine immunization in zebrafish. BMC Genomics 2012 13:319.

\section{Submit your next manuscript to BioMed Central and take full advantage of:}

- Convenient online submission

- Thorough peer review

- No space constraints or color figure charges

- Immediate publication on acceptance

- Inclusion in PubMed, CAS, Scopus and Google Scholar

- Research which is freely available for redistribution 